\author{
Paolo Cavana \\ (professore ordinario di Diritto ecclesiastico nell'Università LUMSA di Roma, \\ Dipartimento di Giurisprudenza, Economia, Politica e Lingue moderne)
}

\title{
Le Sezioni Unite della Cassazione sul crocifisso a scuola: alla ricerca di un difficile equilibrio tra pulsioni laiciste e giurisprudenza europea *
}

The Supreme Court's United Sections on the Crucifix controversy in schools: searching for a difficult balance between laicism and European case-law

\begin{abstract}
This paper examines critically, in the light of the Italian and European case-law, the contents and the juridical arguments of a recent decision issued by the United Sections of the Italian Court of Cassation concerning the Crucifix controversy arisen in a State school. The judges established, on one hand, that the crucifix may be hung in a classroom upon demand of the students as it does not infringe the dissenting teacher's freedom of conscience or of teaching, according to Lautsi ECHR decision (2011); on the other hand, the clash of values involved would need a reasonable accommodation which could require other religious symbols alongside the crucifix or its removal during the lessons of the dissenting teacher. Such a decision, according to the author, appears to be somewhat contradictory and ambiguous, and it does not resolve the case in a well-balanced way.
\end{abstract}

SOMMARIO - 1. Il crocifisso in classe davanti alle Sezioni Unite - 2. Laicità dello Stato e autonomia scolastica - 3 . Conflitto tra diritti e reasonable accommodation 4. La regola della reasonable accommodation nella giurisprudenza straniera e italiana - 5. Il crocifisso come simbolo "essenzialmente passivo" nella giurisprudenza europea - 6. Reasonable accommodation in assenza di una lesione di diritti: rilievi critici - 7. Bilanciamento dei diritti e legislazione scolastica - 8. Il rapporto asimmetrico tra docente e alunni nella scuola: un dato del tutto omesso 9. La rimozione del crocifisso da parte del docente: un atto lecito? Osservazioni conclusive.

\section{1 - Il crocifisso in classe davanti alle Sezioni Unite}

Con la sentenza n. 24414 del 9 settembre 2021 le Sezioni Unite della Cassazione hanno scritto un ulteriore episodio nella sofferta e contrastata 
vicenda del crocifisso nelle aule scolastiche italiane ${ }^{1}$. La decisione sembrerebbe porre un punto fermo su alcuni aspetti della questione, ma ne apre altri sui quali la soluzione proposta non sembra affatto risolutiva e sui quali probabilmente occorrerà tornare. Vi sono poi alcuni punti della decisione non privi di forte ambiguità e destinati a incidere negativamente in ambito scolastico ${ }^{2}$.

La fattispecie nasce, come noto, dal comportamento di un docente di ruolo presso un istituto professionale di Stato, sottoposto a procedimento disciplinare e sanzionato con la sospensione dall'insegnamento per un mese perché, oltre ad avere proferito frasi ingiuriose nei confronti del dirigente scolastico, prima dell'inizio delle sue ore di lezione in una classe dell'istituto, come si legge nella sentenza,

«rimuoveva sistematicamente, in "autotutela", il crocifisso dalla parete dell'aula, per poi riappenderlo al termine delle stesse, così contravvenendo ad una circolare del dirigente scolastico che aveva

\footnotetext{
* Il contributo, sottoposto a valutazione, è destinato alla pubblicazione sulla rivista "Il Diritto Ecclesiastico".

${ }^{1}$ La questione del crocifisso a scuola si colloca sul piano giuridico nell'ambito della più ampia problematica dei simboli religiosi, sulla quale la bibliografia è ormai imponente. A titolo meramente orientativo, e partendo da alcuni più recenti contributi, cfr. S. TESTA BAPPENHEIM, I simboli religiosi nello spazio pubblico. Profili giuridici comparati, Editoriale Scientifica, Napoli, 2019; L. GIANNUZZO, Laicità europea e libertà religiosa alla luce della giurisprudenza della Corte di Strasburgo in tema di simboli religiosi: ipotesi ricostruttive, Libellula Edizioni, Tricase (Le) 2017; L. P. VANONI, Laicità e libertà di educazione. Il crocifisso nelle aule scolastiche in Italia e in Europa, Giuffrè, Milano, 2013; P. CAVANA, I simboli religiosi nello spazio pubblico nella recente esperienza europea, in Stato, Chiese e pluralismo confessionale. Rivista telematica (https://www.statoechiese.it), n. 28 del 2012, pp. 1-47; C. CARDIA, Identità religiosa e culturale europea. La questione del crocifisso, Allemandi \& C., Torino, 2010; AA. VV., Simboli e comportamenti religiosi nella società plurale, a cura di M. PARISI, Edizioni Scientifiche Italiane, Napoli, 2006; AA. VV., I simboli religiosi tra diritto e culture, a cura di E. DIENI, A. FERRARI, V. PACILLO, Giuffrè, Milano, 2006; AA. VV., Symbolon/diabolon. Simboli, religioni, diritti nell'Europa multiculturale, a cura di E. DIENI, A. FERRARI, V. PACILlO, il Mulino, Bologna, 2005; AA. VV., La laicità crocifissa? Il nodo costituzionale dei simboli religiosi nei luoghi pubblici, a cura di R. BIN, G. BRUNELLI, A. PugiotTO, P. VERONESI, Giappichelli, Torino, 2004.

2 Per alcuni primi commenti, cfr. N. COLAIANNI, Dal "crocifisso di Stato" al "crocifisso di classe" (nota a margine di Cass., SS. UU., 9 settembre 2021, n. 24414), in Stato, Chiese e pluralismo confessionale, cit., n. 17 del 2021, p. 17 ss.; P. DUBOLINO, Sezioni Unite e Crocifisso: perché il "ragionevole accomodamento" non convince (September 16, 2021), in www.centrostudilivatino.it; M. GANARIN, Ancora su Cassazione e Crocifisso, fra laicità e reasonable accomodation (September 25, 2021), ivi; A. SALVI, Sezioni Unite e Crocifisso: perplessità sulla regola del caso per caso (September 15, 2021), ivi.
} 
recepito una richiesta di affissione del simbolo proveniente dagli studenti riuniti in assemblea» ${ }^{3}$.

Il docente impugnò sia l'ordine di servizio del dirigente scolastico sia il provvedimento dell'Ufficio scolastico provinciale irrogante la sanzione, e il relativo ricorso, respinto in primo e in secondo grado, era poi arrivato davanti alla Corte di Cassazione, ove la questione era stata rimessa alle Sezioni Unite.

Secondo l'ordinanza di rimessione alle Sezioni Unite, che in parte recepiva le contestazioni del docente ricorrente, l'affissione del crocifisso nell'aula scolastica avrebbe determinato due conseguenze negative: innanzitutto una violazione del principio supremo di laicità dello Stato, in quanto prevista da un regolamento, ossia da un atto amministrativo generale di carattere autoritativo, e pertanto la sua presenza nell'aula scolastica indurrebbe un'identificazione dell'istituzione pubblica con la religione cristiana, di cui il crocifisso è simbolo; in secondo luogo essa produrrebbe una lesione della libertà di insegnamento e di coscienza del docente dissenziente, di cui la circolare del dirigente non avrebbe tenuto conto, e altresì una discriminazione quanto meno indiretta a suo carico, in quanto egli avrebbe subito un "particolare svantaggio" rispetto agli altri docenti per la sua esposizione all'influenza visiva di un simbolo religioso in contrasto con le sue convinzioni, da cui il sorgere di un conflitto tra la libertà del docente e il rispetto della coscienza morale e civile degli alunni che avrebbe richiesto un'opera di bilanciamento dei diritti e libertà coinvolte 4 .

La risposta delle Sezioni Unite alla complessa problematica appare, a mio parere, sostanzialmente corretta sulla prima questione mentre risulta confusa e contraddittoria sulla seconda.

\footnotetext{
3 Cass. civ., sez. un., sent. 9 settembre 2021, n. 24414, p. 2 (§ 1), in www.cortedicassazione.it.

${ }^{4}$ Sull'ordinanza di rimessione alle Sezioni Unite, cfr. P. CAVANA, Il crocifisso davanti alle Sezioni Unite della Cassazione: difesa di diritti o accanimento iconoclasta?, in Stato, Chiese e pluralismo confessionale, cit., n. 14 del 2021, p. 61 ss.; N. COLAIANNI, Il crocifisso di nuovo in Cassazione. Note da amicus curiae, in Stato, Chiese e pluralismo confessionale, cit., n. 12 del 2021, pp. 1; A. LICASTRO, Il crocifisso e $i$ diritti del lavoratore nell'ambiente scolastico (aspettando le Sezioni Unite della Cassazione), in Stato, Chiese e pluralismo confessionale, cit., $\mathrm{n}$. 7 del 2021, pp. 36 ss.; F. VARI, Crocifisso ancora nel mirino, la parola è alla Cassazione, in Avvenire, 23 maggio 2021; J.H.H. WEILER, Verso "Lautsi-bis"? Il crocifisso scolastico (di nuovo) a giudizio, in Forum di Quaderni Costituzionali, 2, 2021, p. 121 ss. (disponibile in www.forumcostituzionale.it): M. TOSCANO, Crocifisso nelle aule scolastiche: una fattispecie inedita al vaglio delle Sezioni Unite, in Quad. dir. pol. eccl., n. 3/2020, p. 887 ss.
} 


\section{2 - Laicità dello Stato e autonomia scolastica}

Dopo un breve richiamo, per la verità non del tutto esatto, all'origine storica della normativa sul crocifisso nelle aule scolastiche italiane - non fu il fascismo a introdurla ma il legislatore risorgimentale (liberale) con il regolamento di esecuzione della laicissima legge Casati sull'istruzione pubblica del $1859^{5}$-, e averne accertato la sua perdurante vigenza con riferimento anche alla scuola media superiore ${ }^{6}$, le Sezioni Unite hanno affermato che la sua imposizione come obbligatoria per effetto di un provvedimento amministrativo di carattere generale risulterebbe in contrasto con il principio di laicità dello Stato, che implica la distinzione degli ordini dello Stato e delle confessioni religiose e l'equidistanza delle pubbliche istituzioni rispetto a tutte le confessioni:

"L'esposizione autoritativa del crocifisso nelle aule scolastiche non è compatibile con il principio supremo di laicità dello Stato. L'obbligo di esporre il crocifisso è espressione di una scelta confessionale. La religione cattolica costituiva un fattore di unità della nazione per il fascismo; ma nella democrazia costituzionale l'identificazione dello Stato con una religione non è più consentita"7.

Tuttavia, precisano i giudici, ciò non implica un divieto assoluto del crocifisso nella scuola pubblica, la cui affissione può essere invece legittimamente ammessa su richiesta degli alunni nell'ambito dell'autonomia delle singole istituzioni scolastiche.

Infatti, si legge nella motivazione,

«deve escludersi che la presenza del simbolo, quando derivi da una richiesta degli studenti in quello spazio pubblico peculiare nel quale essi imparano a convivere insieme e a formarsi culturalmente, qualifichi "tirannicamente" l'esercizio dell'attività che in esso si svolge» ${ }^{8}$.

E si chiarisce:

${ }^{5}$ Per approfondimenti, cfr. L.P. VANONI, Laicità e libertà di educazione, cit., p. 45 ss.; P. CAVANA, La questione del crocifisso in Italia, in www.olir.it, maggio 2004 (oggi in AA. VV., Commenti e contributi di OLIR Osservatorio delle Libertà ed Istituzioni religiose, $1^{\circ}$ Quaderno materiali, Aracne, Roma, 2005, p. 37 ss.).

${ }^{6}$ Cfr. Cass. civ., sez. un., sent. n. 24414 del 2021, cit., pp. 24-28. In questo senso in dottrina si era già espresso A. LICASTRO, Il crocifisso, cit., pp. 52-56.

${ }^{7}$ Cass. civ., sez. un., sent. n. 24414 del 2021, cit., p. 30.

8 Ibidem, p. 41 (§ 14.4). 


\begin{abstract}
"l'esposizione del simbolo è inidonea, tenuto conto del contesto di riferimento, a costituire una forma di proselitismo attivo o di indottrinamento. Il crocifisso non presenta una invasività psicologica tale da condizionare indebitamente il rapporto educativo tra allievi, genitori e istituto scolastico. [...]. La libertà di insegnamento del docente - presidio di pluralismo culturale e di Stato democratico non ne rimane affatto incisa o toccata" 9 .
\end{abstract}

Fin qui il ragionamento segue una sua logica lineare sostanzialmente condivisibile, giungendo a conclusioni già recepite da una parte della dottrina ${ }^{10}$ e sostanzialmente conforme alla giurisprudenza europea:

"La disposizione regolamentare sugli arredi scolastici è suscettibile di esprimere un significato conforme al nuovo contesto costituzionale e alla legislazione che dei principi costituzionali costituisce svolgimento e attuazione, in base ad una interpretazione evolutiva che tramuta l'obbligo di esposizione del crocifisso in una facoltà, affidando alle singole comunità scolastiche la decisione circa la presenza dei simboli religiosi nelle proprie aule"11.

Si potrebbe peraltro osservare che il ricorso al principio di laicità dello Stato, come declinato dai giudici in quello della distinzione degli ordini e dell'equidistanza dello Stato rispetto a tutte le religioni, farebbe presumere l'esclusione assoluta del crocifisso, in quanto simbolo religioso, dalla scuola pubblica ${ }^{12}$. Il fatto che la sua affissione sia prevista da una norma statale o da una delibera degli organi di autonomia scolastica non

\footnotetext{
${ }^{9}$ Ibidem, p. 42 (\$ 14.4).
}

${ }^{10}$ Solo a titolo esemplificativo, cfr. R. BOTTA, Simboli religiosi e autonomia scolastica, in Corriere Giuridico., 2/2004, p. 235 ss., specialmente p. 242: "l'esporre il crocifisso in un'aula scolastica dovrebbe (e non potrebbe che) essere frutto di una scelta positivamente deliberata da ciascuna comunità scolastica"; P. CAVANA, La questione del crocifisso in Italia, cit., p. 58. In argomento da ultimo cfr. A. LICASTRO, Il crocifisso, cit., p. 62 ss., che esamina pro e contro di una simile soluzione anche alla luce della più recente giurisprudenza.

${ }^{11}$ Cass. civ., sez. un., sent. n. 24414 del 2021, cit., p. 35 (§ 12.1).

12 In questo senso è la critica mossa alla sentenza da G. DISEGNI, La Cassazione il crocifisso, un infelice compromesso (in https://moked.it/blog/2021/09/10/la-cassazione-e-il-croci fisso-un-infelice-compromesso/). Tuttavia è da ricordare, tenendo anche conto della provenienza di tali osservazioni, che sull'architrave di tutte le scuole ebraiche dello Stato d'Israele, come in tutte le relative istituzioni statali, è fissato il simbolo religioso ebraico della Mezuzah (cfr. J. H.H. WEILER, Verso "Lautsi-bis"?, cit., p. 122; A ciascuno la sua. La scuola pubblica in Israele. Intervista a Giulia Sarussi Jona, in https://www.hakeillah. com/2_04_07.htm). 
ne mutano infatti la connotazione religiosa e confessionale né la natura di istituzione pubblica della scuola.

In realtà occorre ricordare che il principio di laicità dello Stato, così come delineato nella giurisprudenza costituzionale, è la sintesi di più disposizioni costituzionali, ossia degli artt. 2-3, 7-8, 19 e 20 Cost., ove assume un ruolo centrale "la salvaguardia della libertà religiosa in regime di pluralismo religioso e culturale" (Corte cost., sent. n. 203 del 1989). Sicché pretendere di esaurirne il contenuto in quello della distinzione degli ordini e dell'equidistanza dello Stato rispetto a tutte le religioni, è quanto meno riduttivo. Sotto questo profilo l'argomentazione delle Sezioni Unite sembrerebbe risolversi in una parziale forzatura. Ciò in quanto la laicità dello Stato, nell'esperienza italiana, è del tutto compatibile con una normativa statuale del fenomeno religioso anche all'interno delle istituzioni pubbliche, come nel caso dell'insegnamento religioso nella scuola, che la Corte costituzionale ha giudicato del tutto compatibile con il principio di laicità, a condizione che la sua disciplina, rispondendo a una esigenza diffusa nella popolazione, non leda la libertà di coscienza e di religione di alcuno e non dia luogo a forma alcuna di discriminazione, quindi su base volontaria.

Infatti, come scrisse la Corte, la disciplina dell'insegnamento della religione cattolica nella scuola pubblica

"concorre a descrivere l'attitudine laica dello Stato-comunità, che risponde non a postulati ideologizzati ed astratti di estraneità, ostilità o confessione dello Stato-persona o dei suoi gruppi dirigenti, rispetto alla religione o ad un particolare credo, ma si pone a servizio di concrete istanze della coscienza civile e religiosa dei cittadini"13.

In sostanza, ciò che rappresenta il discrimine nella verifica di compatibilità con il principio di laicità di una disciplina statuale concernente un'attività o simbolo connotato in senso religioso non è tanto la sua fonte normativa o la sua collocazione all'interno di un'istituzione pubblica, come nell'esperienza francese, quanto, in concreto, se essa risponda o meno a una richiesta dei cittadini e se comporti o meno una lesione della libertà di coscienza o di religione o un effetto discriminatorio a danno di qualcuno. Ma si tratta di una precisazione che, per quanto doverosa, non muta la sostanza della decisione dei giudici.

${ }^{13}$ Corte cost., sent. 12 aprile 1989, n. 203, in Foro it., 1989, I-25, c. 1343. 


\section{3 - Conflitto tra diritti e reasonable accommodation}

Il ragionamento svolto dalle Sezioni Unite si fa invece confuso, a mio parere, laddove si sottolinea l'importanza di "un ragionevole bilanciamento" dei diritti e/o valori coinvolti, improntato a criteri di proporzionalità e di ragionevolezza, che i giudici affermano debba essere seguito

«quando vengano in considerazione diverse libertà di coscienza. Occorre evitare che ci sia un tutto per una delle due libertà e un nulla per l'altra, che un diritto si trasformi in "tiranno" nei confronti dell'altro» ${ }^{14}$.

Il ricorso a questo criterio, il così detto "ragionevole accomodamento", che corrisponde alla tecnica del bilanciamento dei diritti praticato nella nostra giurisprudenza costituzionale, visto in opposizione all'applicazione della semplice logica maggioritaria, avrebbe dovuto tradursi, secondo i giudici, nella ricerca condivisa e dialogante di una diversa modalità di esposizione del crocifisso, che tenesse conto anche della posizione del docente dissenziente, rimasto asseritamente estraneo al processo deliberativo, e che dovrebbe includere, secondo i giudici, una serie di possibilità, tra cui una diversa collocazione spaziale del crocifisso sulla parete, il suo affiancamento con altri simboli religiosi o il suo momentaneo spostamento durante la lezione del docente dissenziente ${ }^{15}$.

Ora, si può anche convenire sulla ragionevolezza in astratto di alcune di queste soluzioni, che vorrebbero riflettere il carattere sempre più pluralista, multietnico e plurireligioso della società italiana. Peccato che, da quanto si apprende nella motivazione, nessuno dei protagonisti le aveva concretamente fatte proprie: non il docente, che si è limitato a rimuovere sistematicamente il crocifisso dalla parete durante la sua lezione, né gli studenti in assemblea di classe e gli altri docenti in consiglio di classe, che non avevano sollevato alcuna obiezione all'affissione del crocifisso in aula richiesta dalla maggioranza degli alunni. Pertanto queste indicazioni delle Sezioni Unite possono apparire come un'ulteriore imposizione dall'esterno alla comunità scolastica - non da parte del legislatore né del dirigente scolastico ma dei giudici - in contrasto con la tanto conclamata autonomia delle istituzioni scolastiche, che peraltro oggi rappresenta un principio tutelato a livello costituzionale (art. 117, terzo comma, Cost.).

\footnotetext{
${ }^{14}$ Cass. civ., sez. un., sent. n. 24414, cit., pp. 43-44 (§ 16).

${ }^{15}$ Ibidem, p. 48 (\$ 22).
} 
Ma ciò che, a mio parere, rileva maggiormente, e inficia radicalmente il ragionamento delle Sezioni Unite, è la mancanza nella fattispecie in esame della fondamentale premessa per l'applicazione del criterio di bilanciamento, ovvero la lesione anche solo potenziale di una libertà o di un diritto fondamentale.

Nella motivazione talora si equivoca su questo punto, parlando a volte di conflitto (e bilanciamento) di "diritti", altre volte di conflitto (e bilanciamento) di "valori" alla base della reasonable accommodation, confondendo piani distinti. Sul piano giuridico, quello su cui dovevano pronunciarsi i giudici di legittimità (non di costituzionalità), assume rilievo solo un "conflitto tra diritti", precisi e determinati, o tra interessi di pari rilevanza costituzionale, non un conflitto tra "valori", che come noto sono entità di natura soggettiva e arbitraria, strettamente dipendenti dalle convinzioni e ideologie professate a livello individuale e/o collettivo e di cui si dovrebbe discutere a livello politico e nelle aule parlamentari, non in quelle giudiziarie ${ }^{16}$.

Sotto questo profilo i giudici sembrano avere confuso i due piani, fornendo anche una rappresentazione mistificante, e a tratti grottesca, della regola di maggioranza come oppressiva delle minoranze: ferma la tutela dei diritti fondamentali e del principio di eguaglianza davanti alla legge, che vieta ogni forma di discriminazione, la regola della maggioranza è l'anima della democrazia, che tutti devono accettare come regola prima di ogni forma di pacifica convivenza civile. Se così non fosse, si dovrebbe supporre che tutte le leggi votate dal Parlamento siano oppressive perché frutto inevitabile dell'applicazione della regola della maggioranza. Senza parlare delle stesse decisioni di un collegio di giudici, come le stesse Sezioni Unite, ove spesso si decide il destino di persone e di patrimoni, che sono sempre prese a maggioranza dei loro componenti.

Alla luce di ciò si può rilevare come quella delle Sezioni Unite sia una decisione molto politically correct - ammantata di concetti suadenti come quelli di "neutralità accogliente", "approccio dialogante", "laicità aperta", "soluzione mite" - ma giuridicamente assai fragile, perché viziata da una insanabile contraddizione logica, prima ancora che giuridica, insita nella motivazione.

16 Sui rischi, a livello legislativo e soprattutto giudiziario, di una "tirannia dei valori", che hanno natura soggettiva e carattere tendenzialmente assoluto, refrattario a ogni forma di mediazione o compromesso con altri valori, cfr. C. SCHMITT, La tirannia dei valori. Riflessioni di un giurista sulla filosofia dei valori, a cura di G. GURISATTI, Adelphi, Milano, 2008. 
Infatti da un lato, richiamandosi espressamente alla sentenza Lautsi della CEDU e rigettando le contestazioni del ricorrente, i giudici hanno escluso ogni incidenza dell'esposizione del crocifisso sulla libertà di insegnamento e sulla libertà di coscienza del docente, facendo venire meno anche la tesi della sua rimozione come esercizio di autotutela da parte del pubblico dipendente ${ }^{17}$ e negando ogni risarcimento per una sua asserita discriminazione:

«L'affissione del crocifisso non ostacola il docente nell'esercizio di alcuna delle sue libertà, anche quella di criticare davanti alla classe, in forme legittime e rispettose della altrui coscienza morale, il significato e la stessa presenza del simbolo. La "situazione di particolare svantaggio rispetto ad altre persone" non è ravvisabile nel mero fastidio o nel disaccordo sul piano culturale» 18 .

Dall'altro lato, richiamando alcune pronunce giurisprudenziali straniere, gli stessi giudici hanno accolto la soluzione della reasonable accommodation, che postula invece un conflitto tra diritti, e cioè nella fattispecie una lesione, anche solo potenziale, della libertà di insegnamento e di coscienza del docente derivante dalla presenza del crocifisso nell'aula, arrivando a dichiarare la illegittimità della circolare del dirigente scolastico perché ritenuta viziata dal mancato coinvolgimento dello stesso docente dissenziente nel suo iter decisionale e imponendo modalità alternative di risoluzione della controversia che dovrebbero tenere conto della sua posizione.

Come si possano conciliare queste due tesi, semplicemente giustapposte nella motivazione ma tra loro incompatibili, resta un mistero. La contraddizione è talmente evidente da chiedersi se la motivazione non sia stata scritta da due persone diverse, piuttosto che frutto di una macroscopica svista.

\section{4 - La regola della reasonable accommodation nella giurisprudenza straniera e italiana}

\footnotetext{
17 Anche l'esercizio di poteri di autotutela da parte del pubblico dipendente richiede infatti, come necessario presupposto, la lesione di suoi diritti fondamentali, e quindi costituzionalmente garantiti, cfr. Cass. civ., sez. un., sent. 14 marzo 2011, n. 5924 (§ 7.2) sul caso del giudice Tosti (in https://www.giurcost.org/casi_scelti/Cass.sent.5924-2011.htm).

18 Cass. civ., sez. un., sent. n. 24414, cit., p. 57 (§ 28.2).
} 
La regola della reasonable accommodation, ovvero il bilanciamento dei diritti in gioco, è quella fatta propria, in una fattispecie analoga a quella ivi esaminata, da una nota sentenza del Tribunale costituzionale federale tedesco nel 1995, espressamente richiamata nella motivazione della decisione in esame, che dichiarò l'illegittimità di un regolamento della Baviera nella parte in cui prevedeva l'obbligatoria affissione del crocifisso in tutte le aule delle scuole elementari statali. I giudici tedeschi ritennero che la presenza del crocifisso, simbolo religioso per eccellenza del cristianesimo, determinasse una violazione del principio costituzionale di neutralità dello Stato, in quanto imposto da una disposizione statale, e una lesione della libertà di coscienza degli alunni dissenzienti e dei loro genitori, per l'influsso che tale simbolo era in grado di esercitare sulle coscienze in formazione dei bambini, in contrasto con le loro convinzioni e con quelle dei loro genitori ${ }^{19}$.

La sentenza fu molto attenta nel riconoscere spazio a entrambi gli aspetti della libertà religiosa: la libertà religiosa positiva della maggioranza degli alunni e delle famiglie, conformemente alle tradizioni religiose e culturali del grande Land cattolico, che sostenevano la presenza del crocifisso nell'aula, e la libertà religiosa negativa della piccola minoranza dissenziente, a essa contraria. I giudici ritennero che entrambi gli aspetti andassero doverosamente tutelati, in quanto la neutralità dello Stato apparato non può sopprimere né fare venire meno la libertà degli alunni e dei loro genitori di esprimere le proprie convinzioni religiose e di manifestarle anche in ambito scolastico mediante l'affissione del crocifisso, tanto più in considerazione che il carattere obbligatorio della frequenza scolastica comporta già un sacrificio della loro libertà. Pertanto si trattava di conciliare e di rendere compatibile l'esercizio della libertà religiosa in senso positivo con quella in senso negativo, mediante una procedura decisionale di carattere negoziale che consentisse a tutte le parti di fare valere le proprie ragioni con una riduzione proporzionata delle rispettive pretese, in un clima di tolleranza e rispetto reciproco al fine di superare, in questa delicata materia, la pura applicazione della regola della maggioranza, che avrebbe sacrificato i diritti della minoranza ${ }^{20}$.

19 Per approfondimenti, anche sugli sviluppi normativi seguiti in Germania alla decisione del Tribunale costituzionale federale, cfr. S. CECCANTI, Una libertà comparata: Libertà religiosa, fondamentalismi e società multietniche, il Mulino, Bologna, 2001, p. 222 ss.

${ }^{20}$ In senso favorevole all'applicazione della "soluzione bavarese" anche in Italia, oltre a S. CECCANTI, E se la Corte andasse in Baviera? Relazione introduttiva, in AA. VV., La laicità crocifissa?, cit., p. 1 ss., si espresse, tra gli altri, anche M. CARTABIA, Il crocifisso e il calamaio, ivi, p. 63 ss. 
La decisione, presa all'interno della Corte con una stretta maggioranza, $\mathrm{fu}$ al centro di un acceso dibattito nella dottrina tedesca ${ }^{21} \mathrm{e}$ scatenò vivaci proteste in Baviera, grande Land cattolico in una Germania con una forte tradizione protestante, ma aveva una sua logica, almeno sul piano astratto, fondata sull'accertata lesione della libertà di coscienza degli alunni e delle famiglie dissenzienti. Ancora da venire era l'infuocato dibattito sui simboli religiosi, che avrebbe fortemente impegnato negli anni successivi la giurisprudenza europea, a partire dalla CEDU e dalle alte corti di vari paesi.

Anche la sentenza Multani della Corte Suprema canadese ${ }^{22}$, pure richiamata nella motivazione delle Sezioni Unite, per quanto riferentesi a una fattispecie diversa - il porto del coltello rituale (kirpan) da parte di un alunno di religione sikh -, applica il criterio della reasonable accommodation in presenza di un accertato conflitto, questa volta solo potenziale ma non meno concreto, tra diritti e/o interessi costituzionalmente rilevanti: la libertà del giovane di manifestare anche pubblicamente la propria fede religiosa, mediante il porto del coltello rituale, e la tutela della sicurezza pubblica, cui era ispirato il divieto scolastico di portare con sé armi, anche improprie, come un coltello di determinate dimensioni. In presenza di un simile conflitto, peraltro in un ordinamento di common law, ove è il giudice a creare la norma per il caso concreto, la regola di elaborazione giurisprudenziale della reasonable accommodation appariva correttamente applicata $^{23}$.

Quanto alla citata giurisprudenza della nostra Corte costituzionale, anch'essa è molto chiara ed esplicita nel richiamare l'esigenza di un "bilanciamento tra principi e diritti fondamentali" o "valori", ma da intendersi quest'ultimi non come mere opinioni soggettive bensì sempre come interessi costituzionalmente tutelati, come quello alla salute (art. 32 Cost.) nella nota sentenza sulle acciaierie Ilva di Taranto, e sempre sul presupposto di una loro lesione, reale o potenziale, perché nessuno di questi diritti o interessi possa farsi tiranno sull'altro e determinare il sacrificio o una compressione sproporzionata e ingiustificata di uno di essi ${ }^{24}$.

${ }^{21}$ In argomento, cfr. S. TESTA BAPPENHEIM, I simboli religiosi, cit., p. 458 ss.

${ }^{22}$ Cfr. Cour Suprême du Canada, Multani c. Commission scolaire Marguerite-Bourgeoys, 2 mars 2006, n. 30322 (in https://scc-csc.lexum.com/scc-csc/scc-csc/fr/item/15/index.do).

${ }^{23}$ In argomento, cfr. M. CARTABIA, The Many and the Few: Clash of Values or Reasonable Accommodation?, in American University International Law Review, vol. 33, Iss. 4, 2018, p. 667 ss.

${ }^{24}$ Cfr. Corte cost., sent. 9 aprile 2013, n. 85 (in www.cortecostituzionale.it): "la 


\section{5 - Il crocifisso come simbolo "essenzialmente passivo" nella giurisprudenza europea}

Nel 2011 la Grande Chambre della Corte europea dei diritti dell'uomo, esaminando una vicenda analoga a quella esaminata dal Tribunale costituzionale federale tedesco, è giunta a un esito assai diverso, ribaltando su di un punto fondamentale la premessa di fatto della decisione tedesca: trattandosi di un "simbolo essenzialmente passivo", come tale il crocifisso - hanno affermato i giudici europei a grande maggioranza - non è in grado di esercitare un influsso significativo sulla coscienza in formazione degli alunni, tanto più in un contesto, quello della scuola pubblica italiana, ove l'insegnamento religioso è facoltativo e a ciascuno è dato di esprimere in piena libertà le proprie convinzioni in materia religiosa anche con l'uso di simboli di uso personale e anche manifestando il proprio dissenso verso un simbolo, il crocifisso, sostenuto dalla maggioranza degli studenti 25 .

Costituzione italiana, come le altre Costituzioni democratiche e pluraliste contemporanee, richiede un continuo e vicendevole bilanciamento tra princìpi e diritti fondamentali, senza pretese di assolutezza per nessuno di essi. La qualificazione come "primari" dei valori dell'ambiente e della salute significa pertanto che gli stessi non possono essere sacrificati ad altri interessi, ancorché costituzionalmente tutelati, non già che gli stessi siano posti alla sommità di un ordine gerarchico assoluto. Il punto di equilibrio, proprio perché dinamico e non prefissato in anticipo, deve essere valutato dal legislatore nella statuizione delle norme e dal giudice delle leggi in sede di controllo secondo criteri di proporzionalità e di ragionevolezza, tali da non consentire un sacrificio del loro nucleo essenziale" (Considerato in diritto, § 9).

${ }^{25}$ Cfr. CEDH, Grande Chambre, Lautsi c. Italie (n 30814/06), arrêt, 18 mars 2011. In argomento, tra gli altri, cfr. S. TESTA BAPPENHEIM, I simboli religiosi, cit., p. 285 ss., che con riferimento a tale decisione scrive di uno "spartiacque giurisprudenziale" e "cambio di paradigma"; P. CAVANA, I simboli religiosi, cit., p. 14 ss.; V. TURCHI, La pronuncia della Grande Chambre della Corte di Strasburgo sul caso Lautsi c. Italia: post nubila Phoebus, in Stato, Chiese e pluralismo confessionale, cit., ottobre 2011; M.G. BELGIORNO DE STEFANO, Il crocifisso nelle aule scolastiche in Italia. Una condanna revocata, ma condizionata, dalla Corte Europea dei Diritti Umani, ivi, marzo 2011; M. TOSCANO, La sentenza Lautsi e altri c. Italia della Corte europea di Diritti dell'Uomo, ivi, ottobre 2011; N. HERVIEU, Droit à l'instruction et liberté de religion (art. 2 du Protocole $n^{\circ} 1$ et art. 9 CEDH): Conventionalité de la présence des crucifix dans les salles de classe d'écoles publiques. L'affaire Lautsi c. Italie, ivi, marzo 2011; M. CARTABIA, La Corte del buon senso, in ilsussidiario.net, 21 marzo 2011 (www.ilsussidiario.net); S. FERRARI, Sul crocifisso e su molto altro. Le sentenze della Corte europea dei diritti dell'uomo, in Il Regno. Attualità, 2011, spec. pp. 198-199; L.P. VANONI, La sentenza della Grande Camera sul crocifisso: è una pronuncia corretta, in Quad. cost., XXXI (2011), p. 419 ss.; V. FIORILLO, La sentenza della Grande Camera sul crocifisso: il ritorno del margine di apprezzamento, ivi, p. 422 ss.; S. MANCINI, La sentenza della Grande Camera sul 
La questione, altrettanto importante, dell'identificazione o meno dello Stato con la religione di cui il crocifisso è espressione, cioè il cristianesimo, fu risolta dalla Corte europea ricorrendo alla dottrina del margine di apprezzamento di cui godono gli Stati in materia, riconoscendo l'esistenza tra i paesi aderenti alla Convenzione europea di una pluralità di versioni del principio di laicità. Ma sul punto dell'asserita lesione della libertà di coscienza la Corte è stata assai chiara: l'affissione del crocifisso, in quanto simbolo meramente passivo, nel contesto concreto della scuola pubblica italiana non determina alcuna lesione dei diritti di libertà degli alunni e dei loro genitori dissenzienti. Pertanto non c'è spazio per la soluzione della reasonable accommodation o bilanciamento dei diritti, nemmeno richiamata dalla Grande Chambre nella sua motivazione, poiché tutti i componenti della comunità scolastica sono liberi di esprimere le proprie convinzioni in materia religiosa, anche con l'uso di simboli o in altro modo, rispettando quelle degli altri.

Non c'è da sorprendersi di questo radicale mutamento di approccio: in mezzo - ovvero tra la sentenza della Corte di Karlsruhe (1995) e quella della Grande Chambre della Corte di Strasburgo (2011) - ci sono stati il rapido declino delle ideologie, la crescente secolarizzazione, soprattutto la lunga e controversa querelle sul velo islamico, sorta in Francia ma diffusasi poi in molti altri paesi, che ha indotto a riflettere in ambito giuridico sull'effettiva portata dei simboli religiosi, anche quelli di natura istituzionale, e sulla loro incidenza nella sfera pubblica e nell'ambiente scolastico in particolare. Anche la globalizzazione ha probabilmente giocato un suo ruolo, suscitando in una parte della popolazione europea il recupero di simboli e tradizioni facenti parte della propria identità nazionale, senza contare l'ormai diffuso e dominante analfabetismo religioso, che rende le nuove generazioni quasi indifferenti o del tutto ignare del significato di un simbolo come il crocefisso, e, da ultimo, il grande a rapidissimo sviluppo delle tecnologie informatiche e della comunicazione, che rendono oggi ciascuno di noi bersaglio di un costante flusso di messaggi di ogni tipo tale da renderci in larga misura assuefatti e in qualche modo "protetti" dalla loro influenza.

In effetti, se ci si concentra solo sull'effetto psicologico indotto dalla sua visibilità nell'ambiente scolastico, l'affissione del crocifisso alla parete,

crocifisso: è corretta solo l'opinione dissenziente, ivi, p. 425 ss.; A. BETTETINI, Il crocifisso nelle aule scolastiche: la legittimità di un simbolo che "dà a pensare", in Nuova Giur. Civ. Comm., 6/2011, pp. 281-290; P. TANZARELLA, Le decisioni Lautsi c. Italia: due pesi due misure, in AA. VV., Dieci casi sui diritti in Europa. Uno strumento didattico, a cura di M. CARTABIA, il Mulino, Bologna, 2011, p. 81 ss. 
magari accanto a mappe geografiche o ad altri materiali didattici, è in tutto e per tutto assimilabile ad altri simboli religiosi di uso personale, quali il velo islamico, la kippah ebraica o una collanina al collo con l'immagine di Cristo o della Madonna. Anzi, l'uso di uno di questi simboli, in quanto indossati o portati sulla persona da altri alunni o addirittura dall'insegnante, come del tutto lecito nella scuola italiana, attraggono maggiormente l'attenzione dell'alunno rispetto alla presenza muta di un piccolo oggetto come il crocifisso affisso alla parete.

Tanto è vero che nella giurisprudenza della CEDU solo i simboli di uso personale sono qualificati come "signes extérieures fortes", come tali in grado di condizionare la formazione della coscienza degli alunni, che sarebbero quindi da tutelare contro indebite o abusive pressioni $o$ condizionamenti indesiderati da essi suscitati ${ }^{26}$, mentre il crocifisso è qualificato come simbolo "essenzialmente passivo", che potrebbe porre un problema di impropria identificazione con lo Stato se ne fosse prevista l'affissione obbligatoria ex lege, ma per sé non in grado di produrre, nel contesto pluralista della scuola pubblica italiana, una lesione della libertà di coscienza e di religione dell'alunno dissenziente, libero di esprimere il proprio dissenso o disaccordo rispetto a tale simbolo o ai valori da esso veicolati 27.

In ogni caso le Sezioni Unite non potevano non tenere conto e non applicare nella fattispecie i principi affermati dalla Corte di Strasburgo, che, in forza dell'adesione dell'Italia alla Convenzione europea dei diritti dell'uomo e della nuova formulazione dell'art. 117, primo comma, della Costituzione e, assumono oggi un valore vincolante per il legislatore e, quindi, anche per il giudice.

\section{6 - Reasonable accommodation in assenza di una lesione di diritti: rilievi critici}

${ }^{26}$ Cfr. CEDH, II section, Dahlab c. Suisse (n 42393/98), décision sur la recevabilité, 15 février 2001; CEDH, Grande Chambre, Leyla Sahin c. Turquie ( ${ }^{\circ}$ 44774/98), arrêt, 10 novembre 2005, nn. 113-114. In termini analoghi cfr. CEDH, Ve sez., Kervanci c. France $\left(\mathrm{n}^{\circ}\right.$ 31645/04), arrêt, 4 dicembre 2008, nn. 71-73; CEDH, $V^{a}$ section., Tuba Aktas c. France $\left(\mathrm{n}^{\circ}\right.$ 43563/08), décision sur la recevabilité, 30 juin 2009; CEDH, Bayrak c. France (n 14308/08), Gamaleddyn c. France ( $\left.\mathrm{n}^{\circ} 18527 / 08\right)$, Ghazal c. France $\left(\mathrm{n}^{\circ} 29134 / 08\right)$, J. Singh c. France $\left(\mathrm{n}^{\circ}\right.$ 25463/08), R. Singh c. France (n ${ }^{\circ}$ 27561/08). In argomento, cfr. P. CAVANA, I simboli religiosi, cit., p. 9 ss.

${ }^{27}$ Cfr. CEDH, Grande Chambre, Lautsi c. Italie ( $\left.\mathrm{n}^{\circ} 30814 / 06\right)$, cit. 
La contraddizione nella quale sono incorse le Sezioni Unite, e che ne inficia la motivazione sul piano logico, consiste nell'avere accolto la tesi di un necessario bilanciamento dei diritti (la così detta reasonable accommodation) in assenza della sua essenziale premessa, ovvero l'esistenza di una lesione, reale o anche solo potenziale, di diritti o interessi costituzionalmente rilevanti. Questa lesione è assente - come la stessa Corte ha precisato - perché l'affissione del crocifisso, in quanto simbolo essenzialmente passivo, non è in grado di incidere né di esercitare alcun condizionamento sulla libertà di insegnamento e di coscienza del docente ricorrente, né di produrre una discriminazione a suo carico.

Imporre alla scuola la regola della reasonable accommodation nei dettagliati termini indicati dalla sentenza ${ }^{28}$, in assenza di questo necessario presupposto, equivale a disattendere di fatto la pronuncia della Corte europea e comporta una violazione del principio dell'autonomia scolastica, se non addirittura un'indebita interferenza nelle attribuzioni del legislatore, visto che appena qualche anno fa (2011) le stesse Sezioni Unite della Cassazione avevano affermato, in relazione alla discussa questione dell'affissione del crocifisso nelle aule giudiziarie, pure prevista da una fonte regolamentare, che spettasse solo al legislatore l'opzione in materia tra una laicità per sottrazione (rimozione di ogni simbolo religioso) o una laicità per addizione (aggiunta di altri simboli religiosi accanto al crocifisso) ${ }^{29}$.

${ }^{28}$ In effetti le Sezioni Unite non si sono limitate, in base al principio di laicità, ad affermare il carattere facoltativo, non obbligatorio, dell'affissione del crocifisso, formalmente rimessa a una decisione della scuola, ma pretende poi di determinarne in concreto le specifiche modalità: "l'aula può accoglierne la presenza allorquando la comunità scolastica interessata valuti e decida in autonomia di esporlo, nel rispetto e nella salvaguardia delle convinzioni di tutti, affiancando al crocifisso, in caso di richiesta, gli altri simboli delle fedi religiose presenti all'interno della stessa comunità scolastica e ricercando un ragionevole accomodamento che consenta di favorire la convivenza delle pluralità"(Cass. civ., sez. un., sent. n. 24414, cit., p. 35, § 12.1).

${ }^{29}$ Cfr. Cass. civ., sez. un., sent. 14 marzo 2011, n. 5924 (caso Tosti), ove i giudici ritennero infondata la tesi secondo cui "il rifiuto del ricorrente di tenere udienza poteva ritenersi giustificato dalla mancata autorizzazione a esporre nelle aule giudiziarie la menorah, simbolo della religione ebraica. Per poter accogliere tale pretesa è infatti necessaria una scelta discrezionale del legislatore, che allo stato non sussiste. È vero infatti che sul piano teorico il principio di laicità è compatibile sia con un modello di equiparazione verso l'alto (laicità per addizione) che consenta a ogni soggetto di vedere rappresentati nei luoghi pubblici i simboli della propria religione, sia con un modello di equiparazione verso il basso (laicità per sottrazione). Tale scelta legislativa, però, presuppone che siano valutati una pluralità di profili, primi fra tutti la praticabilità concreta e il bilanciamento dell'esercizio della libertà religiosa da parte degli utenti di un 
La sentenza ivi commentata ha dichiarato l'illegittimità della circolare del dirigente scolastico, che aveva recepito la decisione dell'assemblea di classe - confermata dal consiglio di classe - a favore dell'affissione $\mathrm{dl}$ crocifisso, ritenendo esistente un conflitto tra le ragioni degli alunni, espresse a maggioranza nell'assemblea di classe, e quelle del docente dissenziente, che sarebbe rimasto estraneo al relativo iter decisionale. Ora, a parte il fatto che il ricorrente - come risulta dalla stessa motivazione del provvedimento - ebbe ampio modo di esporre le sue ragioni in quanto membro del consiglio di classe, occorre sottolineare che il confronto tra opinioni diverse e divergenti è cosa del tutto fisiologica in uno spazio aperto al dialogo come la scuola pubblica, e non richiede un meccanismo o regola di bilanciamento imposta dall'esterno: tutti sono liberi di esprimere le proprie opinioni soggettive, anche critiche, e ciascuno è tenuto a rispettare, con spirito di tolleranza, le opinioni contrarie altrui.

Quanto alla regola della maggioranza, tanto bistrattata dai giudici, essa è invece fondamentale in ogni corpo sociale che aspiri all'autogoverno o, quanto meno, a una effettiva, per quanto limitata, autonomia decisionale. Ferma la tutela dei diritti fondamentali e del principio di eguaglianza dei cittadini, l'accettazione di questa regola è essenziale in una democrazia pluralista e ad ogni livello della vita sociale.

Del resto sono gli stessi giudici delle Sezioni Unite a precisare, in un passaggio di una motivazione piena di contraddizioni e di salti logici, che "La regola di maggioranza senza correttivi non può utilizzarsi nel campo dei diritti fondamentali, che è dominio delle garanzie per le minoranze e per i singoli" 30 .

Quindi, al di fuori dei diritti fondamentali, cioè in assenza di una loro lesione, reale o anche solo potenziale, come accertato nella fattispecie in esame, la regola di maggioranza torna a essere l'unico criterio possibile di decisione, che deve essere accettato e rispettato da tutti. Se c'è disaccordo su una determinata questione, prima se ne discute e poi si vota e chi resta in minoranza deve accettare la decisione della maggioranza. Un simile atteggiamento è fondamentale per una effettiva educazione alla

luogo pubblico con l'analogo esercizio della libertà religiosa negativa da parte dell'ateo o del non credente, nonché il bilanciamento tra garanzia del pluralismo e possibili conflitti tra una pluralità di identità religiose tra loro incompatibili" (§ 6.6). In questo senso da ultimo cfr. A. MASOTTI, Crocifisso, il giurista Vari: nella sentenza della Cassazione aspetti positivi e anche ombre, 10 settembre 2021 (in https://www.vaticannews.va/it/ mondo/news/202109/sentenza-cassazione-crocifisso-scuola-cristianesimo-costituzione.print.html)

${ }^{30}$ Cass. civ., sez. un., sent. n. 24414, cit., p. 46 (§ 20). 
tolleranza e al rispetto reciproco, cui sono tenuti a scuola sia gli alunni sia soprattutto i docenti, che dovrebbero dare il buon esempio.

Si noti che la regola della maggioranza è quella che già da tempo disciplina l'affissione del crocifisso nelle scuole italiane. In molte aule scolastiche il crocifisso non è più presente da tempo perché la maggioranza degli alunni e/o delle famiglie era contraria. Non mi pare che simili decisioni abbiano mai indotto qualche docente, magari quello di religione o di altre materie, a ricollocarlo durante le sue lezioni. Un simile comportamento sarebbe qualificato come intollerante e antidemocratico ${ }^{31}$. È quindi curioso che lo stesso comportamento ma di segno contrario ovvero la rimozione del crocifisso attuato da un docente contro la volontà degli alunni - venga invece giustificato dai giudici. Se le opinioni in materia religiosa hanno lo stesso peso, saremmo allora di fronte a una disparità di trattamento a danno dei credenti.

\section{7 - Bilanciamento di diritti e legislazione scolastica}

L'applicazione della regola della reasonable accommodation presuppone un conflitto tra diritti fondamentali o interessi di pari rilevanza giuridica, quindi una lesione - reale o potenziale - di diritti, nella fattispecie espressamente esclusa dai giudici, non una mera differenza di opinioni o di percezioni soggettive.

L'ordinanza di rimessione alle Sezioni Unite aveva recepito in effetti questa impostazione, fondata sull'asserita lesione della libertà di insegnamento e di coscienza del docente dissenziente e sulla sua asserita discriminazione per il "particolare svantaggio" di cui sarebbe stato vittima per effetto della sua esposizione al crocifisso appeso alla parete dell'aula scolastica. Se però si nega la premessa, ovvero l'asserita lesione dei diritti del docente e la sua pretesa discriminazione, come riconoscono i giudici, viene meno anche l'esigenza di un bilanciamento o accomodamento per l'assenza di un conflitto anche solo potenziale tra diritti.

Scorrendo la motivazione della sentenza si scopre peraltro che già dal verbale dell'assemblea di classe e dall'esito del consiglio di classe, di cui faceva parte anche il ricorrente, risultava che la decisione a favore del

\footnotetext{
${ }^{31}$ Cfr. J.H.H. WEILER, Verso "Lautsi-bis"?, cit., p. 126: "Se la maggioranza decidesse di non esporre il crocifisso e il nostro ipotetico sacerdote prima richiamato, entrando nell'aula spoglia, insistesse nell'apporre il crocifisso alla parete, sostenendo che egli non può insegnare senza il crocifisso, meriterebbe di essere sospeso al pari dell'insegnante laico che non ha voluto insegnare con il crocifisso esposto".
} 
crocifisso non aveva creato alcun problema tra gli alunni e tra gli altri docenti. Quindi il ricorrente non rappresentava eventuali altri alunni o docenti dissenzienti ma solo sé stesso.

Anche l'interpretazione della legislazione scolastica ${ }^{32}$ fatta propria dalle Sezioni Unite riflette questa contraddizione di fondo. Nella prima parte della motivazione la libertà di insegnamento del docente e la tutela della coscienza morale degli alunni vengono poste sullo stesso piano, facendone derivare l'esigenza di un loro bilanciamento, secondo quella che per i giudici sarebbe una "interpretazione costituzionalmente orientata" di tale disposizione. Nella seconda parte, invece, si riconosce più correttamente - e in modo aderente alla formulazione legislativa - che la tutela della libertà di coscienza degli alunni è piuttosto un limite alla libertà di insegnamento del docente:

"Nella fattispecie, che qui ci occupa, della affissione derivante da una richiesta degli studenti, la mera percezione visiva del crocifisso è il risultato dell'esercizio di un diritto fondamentale da parte degli alunni che fanno parte della stessa comunità. Non è configurabile discriminazione per il fatto che il docente non è stato risparmiato, nello spazio pubblico condiviso, da quella esposizione e da quella percezione visiva. Lo spirito di tolleranza e il rispetto della coscienza morale degli alunni, cui il docente è tenuto a conformare il suo comportamento, valgono proprio a fronte di opinioni o convinzioni da lui non condivise" ${ }^{\prime 33}$.

In sostanza il bilanciamento tra differenti diritti e libertà in questo caso risulta già stabilito in modo chiaro e conforme ai principi costituzionali dal legislatore, sicché i giudici di legittimità dovevano limitarsi a prenderne atto.

\section{8 - Il rapporto asimmetrico tra docente e alunni nella scuola: un dato del tutto omesso}

Un ulteriore limite della sentenza, presente anche nell'ordinanza di rimessione alle Sezioni Unite, è che in essa non si tiene conto in alcun modo del rapporto asimmetrico che intercorre tra docente e alunni nella scuola: il primo in posizione di superiorità didattica e disciplinare e libero,

32 Cfr. artt. 1-2 d. lgs. 16 aprile 1994, n. 297 - Testo unico delle disposizioni legislative vigenti in materia di istruzione, relative alle scuole di ogni ordine e grado.

33 Cass. civ, sez. un., sent. n. 24414 del 2021, cit., p. 58 (§ 28.3). 
nella sua attività, di conformare il suo insegnamento impartito agli alunni alle proprie convinzioni ideologiche e/o religiose e di condizionare con ciò la formazione della personalità gli alunni, soggetti minori in età di formazione, che sono invece in posizione di soggezione e di subordinazione disciplinare e didattica nei confronti del docente.

Per essi l'affissione del crocifisso, come pure l'esposizione di altri simboli di uso personale, può essere l'unico modo per fare valere e manifestare liberamente, cioè senza esporsi a rappresaglie del docente, le proprie convinzioni in materia religiosa. Senza contare che il comportamento del docente potrebbe urtare anche contro le responsabilità educative e la libertà di coscienza dei genitori degli alunni (cfr. art. 30, primo comma, Cost.).

$\mathrm{Su}$ questo punto era intervenuta in termini molto chiari la Grande Chambre della Corte europea nella sentenza Lautsi, la quale, nel qualificare il crocifisso come un simbolo meramente passivo e inoffensivo, ne aveva rimarcato la differenza proprio rispetto a un discorso didattico, cioè alla lezione di un docente, sempre in grado di condizionare attivamente la coscienza degli alunni:

"le crucifix apposé sur un mur est un symbole essentiellement passif, et cet aspect a de l'importance aux yeux de la Cour, eu égard en particulier au principe de neutralité [...]. On ne saurait notamment lui attribuer une influence sur les élèves comparable à celle que peut avoir un discours didactique ou la participation à des activités religieuses" 34 .

Si noti come un simile approccio, volto a tutelare prioritariamente la libertà di coscienza degli alunni contro possibili abusi della libertà di insegnamento del docente, corrisponda esattamente a quanto prevede la nostra legislazione scolastica, laddove pone il rispetto della coscienza morale e civile degli alunni come limite alla libertà di insegnamento (art. 1, primo comma, D. lgs. n. 297 del 1994).

Discostandosi da questo principio, equilibrato e del tutto condivisibile, la sentenza delle Sezioni Unite fa invece prevalere la posizione del docente sull'intera comunità scolastica, di cui egli non è parte su un piano di parità, smentendo l'immagine della scuola pubblica come luogo di confronto e di libero sviluppo della personalità degli alunni ${ }^{35}$.

${ }^{34} \mathrm{CEDH}$, Grande Chambre, Lautsi c. Italie ( $\left.{ }^{\circ} 30814 / 06\right)$, cit., $§ 72$.

${ }^{35}$ Si noti che l'art. 1 del d. lgs n. 297 del 1994, cit., non si limita a prevedere la tutela della coscienza morale e civile degli alunni come limite alla libertà di insegnamento del 
Come già precisato, la stessa legislazione scolastica fa del "rispetto della coscienza civile e morale degli alunni" un limite alla libertà di insegnamento del docente, non li pone sullo stesso piano, come invece sostiene la sentenza per desumerne l'esigenza di un loro bilanciamento, che invece è già previsto dal legislatore in termini di chiara subordinazione della seconda alla prima, ossia la libertà di coscienza degli alunni. Per cui l'interpretazione che di tali disposizioni forniscono le Sezioni Unite non è "costituzionalmente orientata", come da loro sostenuto, ma tende piuttosto a manipolare il chiaro significato della disposizione, fornendo una rappresentazione del rapporto docentestudenti - del tutto sbilanciata a favore del primo anche al di fuori della sfera didattica e disciplinare - che non corrisponde alla funzione della scuola e al suo impegno a rispettare la libertà di coscienza (e di religione) degli alunni e dei loro genitori.

Sotto questo profilo si può rilevare come la regola dell'accomodamento ragionevole, se anche fosse praticabile - ciò che nella fattispecie non è per la mancanza di un conflitto tra diritti -, andrebbe comunque applicata non in astratto ma tenendo sempre conto del contesto concreto, cioè della scuola pubblica italiana, nella quale, come puntualmente rilevato dalla CEDU, ciascuno può esprimere liberamente le proprie convinzioni in materia religiosa, anche mediante l'uso di simboli di altre fedi, e nel quale il docente riveste una posizione di superiorità, sul piano didattico e disciplinare, che gli consente non solo di esprimere sempre, mediante la sua attività di insegnamento, le proprie convinzioni ma anche di imporle di fatto agli studenti, laddove questi ultimi sono in posizione di mera soggezione, esposti al potere valutativo e disciplinare del primo.

Senza contare che la soluzione delle Sezioni Unite, esclusa ogni connotazione confessionista della presenza del crocifisso in quanto richiesta dagli alunni, sembrerebbe richiedere a questo punto l'applicazione della regola dell'accomodamento anche per i simboli religiosi di uso personale, come nel caso Multani ${ }^{36}$, soprattutto per quelli eventualmente portati o indossati dal docente, che rappresenta

docente, ma prevede altresì che "l'esercizio di tale libertà è diretto a promuovere, attraverso un confronto aperto di posizioni culturali, la piena formazione della personalità degli alunni" (art. 1, secondo comma).

${ }^{36}$ Cfr. Cour Suprême du Canada, Multani c. Commission scolaire Marguerite-Bourgeoys, cit., decisione espressamente richiamata nella motivazione della sentenza delle Sezioni Unite. 
l'istituzione scolastica, poiché anch'essi possono determinare un "conflitto tra valori" all'interno della classe.

\section{9 - La rimozione del crocifisso da parte del docente: un atto lecito? Osservazioni conclusive}

Da ultimo si può osservare che, seguendo la logica adottata dalle Sezioni Unite, il comportamento del docente risultava comunque censurabile, non solo per le espressioni ingiuriose rivolte al dirigente scolastico, ma perché egli per primo non aveva dimostrato alcuna disponibilità all'ascolto degli alunni né cercato un ragionevole accomodamento con gli stessi - come invece avrebbe dovuto per le sue primarie responsabilità educative e la sua posizione di autorità -, rimuovendo sic et simpliciter con un atto di forza il crocifisso dalla parete dell'aula durante le sue lezioni e seguitando a farlo nonostante il parere contrario espresso dagli alunni, senza mai provare a entrare in dialogo con la classe e creando un clima di tensione e di scontro. Comportamento tanto più grave in quanto, come è stato poi accertato dai giudici, la presenza del crocifisso non ledeva in alcun modo la sua libertà di insegnamento e di coscienza né lo sottoponeva ad alcuna discriminazione.

Pertanto la rimozione del crocifisso, ripetutamente attuata dal docente, non poteva certo qualificarsi come esercizio di autotutela - che, come precisato dalla stessa Cassazione, richiede una lesione dei diritti fondamentali del pubblico dipendente ${ }^{37}$ - ma semplicemente come un abuso di autorità, che sembra evocare anche un certo dogmatismo di matrice laicista ${ }^{38}$.

\footnotetext{
${ }^{37}$ Cfr. Cass. civ., sez. un., sent. n. 5924 del 2011, cit.

${ }^{38}$ Come i credenti, anche i non credenti corrono a volte il rischio di cadere in forme di dogmatismo, che si rivelano come tali nel momento in cui tendono a imporre forzatamente le proprie opinioni agli altri, come in questo caso, in cui il docente, abusando della sua posizione di autorità all'interno della classe, rimuoveva sistematicamente il crocifisso dall'aula durante le sue lezioni nonostante il parere contrario espresso dagli alunni. Mi piace richiamare in proposito un passaggio di un famoso scritto sulla scuola di Gaetano Salvemini (G. SALVEMINI, Che cos'è la laicità, in Opere, vol. V, Scritti sulla scuola, a cura di L. BORGHI e B. FINOCCHIARO, Feltrinelli, Milano, 1966, pp. 881-896), tra i più illustri esponenti della cultura laica italiana: "Ognuno di noi ha la sua fede: ebraica, o cattolica, o protestante, o atea, o agnostica; e nulla deve costringere a mutilare noi stessi o dissimulare le nostre convinzioni. Ma nessuno di noi si crede in diritto di imporre la sua credenza agli alunni. Nessuno di noi prende pretesto dal suo insegnamento per fare della propria fede propaganda settaria. E se qualcuno
} 
Un simile comportamento che, sulla base di quanto riportato nella motivazione, può apparire quanto meno provocatorio e causa di tensioni nella classe, viene invece in qualche modo giustificato dai giudici, che hanno imputato la responsabilità del mancato dialogo al solo dirigente scolastico e non al docente dissenziente, che avrebbe invece dovuto per primo adottare un atteggiamento dialogante verso gli alunni invece di fare valere arbitrariamente la sua posizione di autorità rimuovendo sistematicamente il crocifisso dall'aula durante le lezioni: non proprio un esempio di reasonable accommodation e di disponibilità al dialogo ${ }^{39}$.

$\mathrm{Su}$ questo punto la decisione delle Sezioni Unite risulta estremamente ambigua, poiché sembrerebbe conferire al docente una sorta di "licenza di rimozione" (del crocifisso), pur in assenza di ogni lesione di suoi diritti e contro la volontà degli stessi alunni e genitori, che, oltre a una serie di inconvenienti pratici ${ }^{40}$, rischia di essere fonte di nuove tensioni e di forzature all'interno della scuola, vanificando lo stesso ricorso al dialogo invocato dai giudici. C'è poi da chiedersi se la stessa soluzione verrebbe applicata anche nell'ipotesi, eguale e contraria, in cui un docente ricollocasse il crocifisso durante le sue lezioni dopo che gli alunni a maggioranza ne avessero ottenuto la rimozione, come già avvenuto in molte scuole dell'Italia settentrionale. Questa sola ipotesi ci fa intendere le implicazioni e le ulteriori tensioni che la decisione dei giudici rischia di suscitare nell'affrontare simili questioni.

La verità è che quella dei simboli religiosi è materia da trattarsi con estrema attenzione ed equilibrio nelle democrazie pluraliste e multiculturali del nostro tempo, poiché su di essa si misura il livello di tutela effettiva assicurata al diritto di libertà religiosa in ciascun ordinamento, come ha già ampiamente dimostrato l'infuocato dibattito sul velo islamico e il caso del crocifisso davanti alla Corte di Strasburgo (caso

venisse meno a questo dovere di lealtà, tutti saremmo concordi nel chiedere la sua espulsione dalla scuola".

39 Molto severo, ma con buone ragioni, sul comportamento del docente nella fattispecie in esame, peraltro in singolare sintonia con il pensiero del laico Salvemini, risulta J.H.H. WEILER, Verso "Lautsi-bis"?, cit., p. 126: "La condotta dell'insegnante mira a reintrodurre uno Stato confessionale, la cui religione è la laicità imposta a tutti, allo stesso modo in cui un tempo s'imponeva la cristianità. Docenti di questo tipo non dovrebbero trovare spazio in una scuola pluralista. In essa si dovrebbe, infatti, insegnare rispetto per i laici, se una classe decidesse di esporre il crocifisso, e rispetto per i credenti, se una classe votasse di non averlo. Il primo è l'atteggiamento di chi pensa che il proprio diritto conti più di quello degli altri e che vuole imporlo violando il diritto altrui; mentre il secondo è l'atteggiamento democratico, liberale e pluralista".

${ }^{40}$ Sui quali opportunamente cfr. A. LICASTRO, Il crocifisso, cit., pp. 67-68. 
Lautsi) $^{41}$ : ma sembra che questo aspetto non sia stato del tutto compreso dai nostri giudici.

Sorprende anche il curioso atteggiamento di alcuni commentatori, sempre pronti a stracciarsi le vesti per la (doverosa) tutela della libertà di coscienza dei non credenti, e in questo caso invece assertori della sostanziale irrilevanza della questione, come se la libertà in materia religiosa avesse un diverso peso a seconda che sia fatta valere da credenti o da non credenti.

Concludendo, a me pare che le numerose contraddizioni e ambiguità di questa pronuncia, redatta forse in modo affrettato e senza adeguato approfondimento, siano tali da rendere a questo punto auspicabile un intervento del legislatore sulla delicata questione che recepisca i principi affermati dalla Corte europea dei diritti dell'uomo (sent. Lautsi), come sempre accaduto in altri ambiti, e consenta poi eventualmente alla nostra Corte costituzionale, giudice dei diritti, di pronunciarsi in merito ${ }^{42}$. Con una decisione che, tenendo conto delle istanze della comunità scolastica, renda finalmente giustizia a un simbolo che più di qualsiasi altro incarna le migliori tradizioni - di profonda umanità e solidarietà - del nostro paese, al di là di ogni differenza di credo, e che ha ispirato nel corso dei secoli le più alte espressioni dello spirito e dell'arte italiane.

41 Oltre alla bibliografia già citata, cfr. G. DALLA TORRE, Simboli religiosi e libertà religiosa, in Nuovi Studi politici, 1-2, 2005, p. 137 ss.

42 In senso favorevole a una soluzione legislativa della questione, all'epoca (2004) in particolare esemplata sul modello bavarese, si era già espressa una parte autorevole della dottrina (tra gli altri cfr. S. CECCANTI, E se la Corte andasse in Baviera?, cit., e M. CARTABIA, Il crocifisso e il calamaio, cit.). Più di recente, sulla possibilità di "aggiornamenti normativi che possano mutare tale quadro e sciogliere i dubbi oggi esistenti", si è espresso, poco prima della decisione delle Sezioni Unite, A. LICASTRO, Il crocifisso, cit., p. 67. 